\title{
Controllable preparation and high properties of fluorescence and surface enhanced raman spectra encoded poly(glycidyl methacrylate) microsphere
}

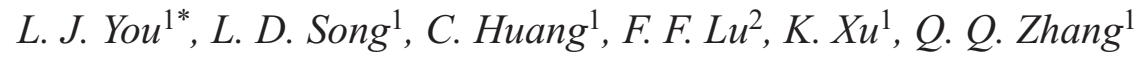 \\ ${ }^{1}$ Institute of Biomedical and Pharmaceutical Technology, Fuzhou University, 350002 Fuzhou, China \\ ${ }^{2}$ College of Chemistry, Fuzhou University, 350108 Fuzhou, China
}

\begin{abstract}
Novel fluorescence and SERS encoded microspheres based on poly(glycidyl methacrylate) (PGMA) microspheres were developed by a controllable approach. The microspheres were encoded by four fluorescence dyes and three surface enhanced Raman spectra (SERS) probes. Multiple dyes could be simultaneously incorporated into the PGMA microspheres for encoding and the intensity of the fluorescence can be tuned by adjusting the feeding ratio. The PGMA microspheres were coated by silver nanoparticles and encoded by single and multiple SERS reporters. The fluorescence and SERS joint encoded PGMA microspheres were fabricated. All the fluorescence encoding signals can be excited at $488 \mathrm{~nm}$. The fluorescent and SERS signals of dual-mode microspheres could be obtained from two optical channels and non-overlapping of emission spectra was observed in these encoded microspheres, which greatly increased the encoding capacity. The as prepared fluorescence and SERS encoded microspheres possess stable and distinct spectral encoding signals, large encoding capacity.
\end{abstract}

Keywords: polymer composites, polymer microsphere, spectra encoding

\section{Introduction}

In the past decades, the microsphere-based suspension array technology shows a particularly important role in labeling, detection, and high throughput screening [1-3]. Encoded microspheres based suspension arrays have many advantages including fast binding kinetics, flexible array preparation, and multiplexed analytical capability over traditional planar array technology. The encoded microspheres have large specific surface area and small volume, providing more molecular binding sites, and thus have higher sensitivity $[4,5]$. In the analysis of the capacity and speed, the microsphere-based arrays rely on the specific probe molecules of different encoded microsphere to analyze and detect multiple analytes simultaneously [6]. The key problem of the suspension array is the preparation of reliable and stable encoded microspheres, which determines the encoding signal, capacity and various chemical properties of the microspheres.

Current encoding methods of microspheres include spectral encoding $[7,8]$, chemical encoding $[9,10]$, physical encoding $[11,12]$ and pattern encoding [13]. As a promising encoding method, spectral encoding includes fluorescence encoding [14, 15], Raman spectra encoding $[16,17]$, and photonic-crystal encoding $[4,18]$. As the most studied encoding method, the fluorescence encoding has the advantages of high encoding capacity, stable signal and easy identification $[3,19]$. Many methods have been developed for the preparation of fluorescence encoded microspheres including adsorption method [20], embedding method $[21,22]$, and self-assembly method [23, 24]. Among these, the embedding technology is considered as

$\overline{{ }^{*} \text { Corresponding author, e-mail: yljyoyo@126.com }}$

(C) BME-PT 
simple, uneasy to leak and suitable for the preparation of microspheres with multi-fluorescence emission. However, fluorescent encoding exhibits shortcomings including spectral overlap, energy transfer, photo bleaching and these result in a decrease of the encoding capacity $[25,26]$. In recent years, surface enhanced Raman spectrum (SERS) has been reported due to its high sensitivity, high specificity and unique microanalysis capability [27, 28]. SERS can be used to detect the unique Raman 'fingerprint' information of small target molecules with specific chemical groups through a relatively simple equipment with convenient operation. The SERS phenomenon can be observed in analytes adsorbed on the surface of crude noble metal particles (such as Ag, $\mathrm{Au}$ or $\mathrm{Cu}$ ) [29]. The phenomenon is mainly caused by electromagnetic enhancement mechanism and the enhancement strongly depends on the distance between adsorbed molecules and the surface of plasmonic materials [30]. Silver, as a classical SERS substrate, has a strong localized surface plasmon resonance effect, it can cover the wavelength range in which most Raman measurements occurred [27]. Nevertheless, it is currently the only way capable of simultaneously detecting a single molecule and providing its fingerprint chemical information. Theoretically, the coding capacity of the encoded microspheres can be calculated according to the formula: $C=N^{\mathrm{m}-1}$, where $C$ is the number of coding, $N$ is the number of intensity levels and $\mathrm{m}$ is the number of encoding elements [5]. However, in the practical studies, the doping of the encoding elements (e.g., fluorophores, quantum dot) may affect the performance of the microspheres, and the spectral overlap and interference between encoding elements can significantly reduce the actual coding capacity [25]. To overcome the above drawbacks of the spectral overlap and increase the encoding capacity of the microbeads approach, we developed reliable and stable SERS and fluorescent dual model encoded microspheres in our previous studies based on phenolic resin and melamine resin microspheres through condensation polymerization $[5,26]$. In this work, we aimed to develop a new SERS and fluorescent dual model encoded microspheres by chain polymerization using the poly(glycidyl methacrylate) (PGMA) microspheres as the microsphere material, which is rich in epoxide groups and beneficial for immobilization of fluorescent molecules [31]. The PGMA microsphere was designed for the fluorescence and surface enhanced Raman spectra joint encoding herein for the first time. Second, we expanded the SERS and fluorescent dual model encoding from the condensation polymerization materials in our previous studies $[5,26]$ to chain polymerization material. Third, the PGMA microspheres are rich in epoxide groups and beneficial for immobilization of fluorescence dye molecules and the as prepared fluorescent microspheres possessed stable strong fluorescence characteristics. Moreover, the fluorescent encoded FGMA microspheres were modified with Ag nanoparticles and Raman label molecules for combination of SERS encoding, which is also rarely reported.

Herein, a new fluorescence and SERS encoded microspheres based on PGMA was designed and developed, which combined the advantages of fluorescent encoding and Raman encoding. PGMA were fabricated by dispersion polymerization and encoded by four fluorescence molecules and three SERS probes. The as prepared microspheres were uniform in size. Moreover, the encoded microspheres have strong fluorescent emissions and SERS coding signals, and non-overlapping of emission spectra was observed in these encoded microspheres, which is rarely reported in other reports. A silica shell was coated on the surface of the encoded microspheres for the benefit of enhanced biocompatibility and further application. The as prepared fluorescence and SERS encoded microspheres possess stable spectral encoded signal, large encoding capacity.

\section{Experimental section}

\subsection{Materials}

Glycidyl methacrylate (GMA, 98\%), polyvinylpyrrolidone (PVP-K30, molecular mass 40000), ethanol (EtOH, $100 \%)$, silver nitrate $\left(\mathrm{AgNO}_{3}, 99.8 \%\right)$ and tetraethyl orthosilicate (TEOS) were purchased from Sinopharm Chemical Reagents Co., Ltd., Shanghai, China. Aqueous ammonia solution $\left(\mathrm{NH}_{3} \mathrm{H}_{2} \mathrm{O}\right.$, 25\%), azo-diisobutyronitrile (AIBN, 98\%), Rhodamine B (RhB, 95\%), Phloxine B (PhB, 95\%), Sulforhodamine 101 (SR101, 98\%) and Fluorescein isothiocyanate (FITC, 98\%) were purchased from Shanghai Chemical Reagent Co., Ltd., Shanghai, China. Raman reporter molecules (XBT) including 4-aminobenzenethiol (4-ABT, $\mathrm{C}_{6} \mathrm{H}_{7} \mathrm{NS}, 98 \%$ ), 4chlorobenzenethiol (4-CBT, $\mathrm{C}_{6} \mathrm{H}_{5} \mathrm{ClS}, 98 \%$ ), 4-hydroxybenzenethiol (4-HBT, $\mathrm{C}_{6} \mathrm{H}_{6} \mathrm{OS}, 98 \%$ ), $n$-butylamine $\left(\mathrm{C}_{4} \mathrm{H}_{11} \mathrm{~N}, 99 \%\right)$ were purchased from Aladdin Chemical Reagent Co., Ltd., Shanghai, China. 


\subsection{Synthesis of PGMA and fluorescent PGMA (FPGMA) microspheres}

The PGMA microspheres and fluorescent poly(glycidyl methacrylate) microspheres (FPGMA) were fabricated by dispersion polymerization. Typically, $50 \mathrm{mg}$ fluorescent molecules (FITC, RhB, PhB and SR101) were dissolved in $100 \mathrm{ml}$ deionized water to form fluorescent stock solutions. 1.0 g PVP-K30 was dissolved in a mixed solvent of ethanol $(87 \mathrm{ml})$ and deionized water $(13 \mathrm{ml})$ in a three-necked flask. Then, $3 \mathrm{ml}$ fluorescent solution was added to the mixture and stirred until blended. Thereafter, nitrogen was filled into to the flask and $5.0 \mathrm{~g}$ GMA containing $0.1 \mathrm{~g}$ AIBN were injected into the mixture. Then, and the mixture was heated to $70^{\circ} \mathrm{C}$ and the polymerization reaction proceeded for $12 \mathrm{~h}$ in nitrogen. After the reaction was completed, the mixture was cooled to room temperature. The obtained FPGMA microspheres were collected by centrifugation and washed by $50 \mathrm{ml}$ ethanol and $50 \mathrm{ml}$ deionized water for three times, respectively. Finally, the microspheres were dried at $40^{\circ} \mathrm{C}$ under vacuum $(-0.06 \mathrm{MPa})$ for $24 \mathrm{~h}$ and the yield of the products was $76 \%$. The preparation procedure of PGMA microspheres was the same as those of FPGMA, but without the addition of fluorescent molecules. The yield of the PGMA was $87 \%$.

\subsection{Preparation of PGMA/Ag}

(Ag nanoparticles coated PGMA) and FPGMA/Ag (Ag nanoparticles coated FPGMA) microspheres

The PGMA and FPGMA microspheres were coated with Ag nanoparticles through in situ-deposition method. Typically, $20 \mathrm{mg}$ PGMA or FPGMA microspheres were dispersed in the $30 \mathrm{ml} \mathrm{AgNO}_{3}$ ethanol solution. Then n-butylamine (the dosage ratio of nbutylamine to $\mathrm{AgNO}_{3}$ was $2.5: 1 \mathrm{ml} / \mathrm{g}$ ) was added into the solution and the mixture was treated by ultrasonication for $5 \mathrm{~min}$. After that, the mixture was heated to $50^{\circ} \mathrm{C}$ and reacted for $2 \mathrm{~h}$. Finally, the obtained PGMA/Ag and FPGMA/Ag microspheres were collected by centrifugation, washed with $30 \mathrm{ml}$ ethanol and $30 \mathrm{ml}$ deionized water three times, and dried at $40^{\circ} \mathrm{C}$ under vacuum for $4 \mathrm{~h}$.

\subsection{Modification of PGMA/Ag and FPGMA/Ag composite microspheres with Raman reporters}

One or two Raman reporter molecules XBT, including 4-ABT, 4-CBT or 4-HBT, were adsorbed onto
PGMA/Ag and FPGMA/Ag microspheres for SRES encoding. Specifically, $1.0 \mathrm{mg}$ PGMA/Ag or FPGMA/ $\mathrm{Ag}$ microspheres was dispersed in $2 \mathrm{ml}$ ethanol containing $0.4 \mathrm{mg}$ XBT. After shaken and reacted at room temperature for $2 \mathrm{~h}$, the SERS encoded PGMA/ Ag (PGMA/Ag-XBT) and the SERS encoded FPGMA/Ag (FPGMA/Ag-XBT) microspheres were obtained by centrifugation and washed with $30 \mathrm{ml}$ ethanol and $30 \mathrm{ml}$ deionized water three times to remove the excess XBT.

\section{5. $\mathrm{SiO}_{2}$ coating of $\mathrm{FPGMA} / \mathrm{Ag}-\mathrm{XBT}$ microspheres}

In order to facilitate the further surface modification of the microspheres and protect it from outside interference, the Raman reporter modified FPGMA/AgXBT microspheres was coated with a thin silica layer by a modified Stöber method [32]. Typically, $20 \mathrm{mg}$ FPGMA/Ag-XBT were dispersed in a mixture of $20 \mathrm{ml}$ water and $60 \mathrm{ml}$ ethanol. Then $2 \mathrm{ml}$ ammonium hydroxide (concentration 25\%) were injected into the dispersion and treated by ultrasonic for $5 \mathrm{~min}$. Thereafter, $2 \mathrm{ml}$ TEOS were added into the homogenized system and reacted for $6 \mathrm{~h}$ under stirring. Finally the silica coated composite microspheres (FPGMA/Ag-XBT/SiO2) were obtained by centrifugation.

\subsection{Characterization}

Scanning electron microscopy (SEM) was investigated using a scanning electron microscope (Tecnai G2 F20 S-Twin, FEI, USA). The fluorescence photo micrographs were obtained from OLYMPUS inverted fluorescence microscope (IX53, Japan). The fluorescence spectra were detected using the HORIBA fluorescence spectrometer (FluoroMax-4, France). Fourier transform infrared spectra (FT-IR) were obtained on a FT-IR spectrometer (NEXUS-870, USA). The spectra widths are typically over the range of $4000-400 \mathrm{~cm}^{-1}$. Thermogravimetric analysis (TGA) measurements were performed on a TGA instrument (STA-449C, Germany) with a heating rate of $10{ }^{\circ} \mathrm{C} / \mathrm{min}$ and taken under a constant flow of nitrogen of $40 \mathrm{ml} / \mathrm{min}$. Powder X-ray diffraction (XRD) patterns were obtained using a X'Pert Pro (Panalytical, Netherlands) diffraction meter with $\mathrm{Cu} \mathrm{K} \alpha$ radiation $\lambda=0.154 \mathrm{~nm}$ operating at $40 \mathrm{kV}$ and $40 \mathrm{~mA}$. Transmission electron microscopy (TEM) analyses were taken on a Transmission Electron Microscope transmission electron microscope 
(JEOL JEM-2100F, USA) with an accelerating voltage of $80 \mathrm{kV}$. SERS spectra were measured using the HORIBA spectrometer (XploRA, France) with $632.8 \mathrm{~nm}$ laser excitation. The SERS measurements were carried out by focusing the laser on microsphere samples.

\section{Results and discussion}

\subsection{Synthesis and characterization of fluorescent microspheres}

The preparation route of the encoded FPGMA/Ag$\mathrm{XBT} / \mathrm{SiO}_{2}$ microspheres was shown in Figure 1. In general, the sizes, morphologies and dispersity of microspheres are important for preparation and application. Thus, the synthesis of PGMA microspheres was first investigated. The controllable synthesis of PGMA with tunable microspheres sizes could be achieved by adjusting the dosage of PVP-K30 and ratio of ethanol to water. When the dosage of PVPK30 decreased from 3.0 to $0.5 \mathrm{~g}$, the sizes of PGMA microspheres increased from 1.2 to $2.4 \mu \mathrm{m}$. In the formation process of PGMA microspheres, PVP-K30 acted as nucleation sites and improved the stability of for the microspheres. With the dosage of PVP$\mathrm{K} 30$ decreased, the nucleation sites reduced and resulted in the increase of microsphere size. When the ratio of ethanol to water varied from 95:5 to $85: 15$, the sizes of microspheres increased from 0.8 to $4.78 \mu \mathrm{m}$. Ethanol is superior to water for the dispersion of GMA monomers and initial polymer chains, which is more conducive to the stability of the particles. With the decrease of the ratio of ethanol to water, the dispersion of initial segments and particles became worse, causing more aggregation of the initial particles and less nucleation particles, thus resulted in the increase in sizes of the microspheres. The morphology and size distribution of the obtained microspheres were characterized by SEM and the polydispersity (PDI) of the microspheres was listed in Table 1. From Figure 2 and Table 1, the as prepared microspheres are uniform in size. It was observed the PDI of the PGMA was lowest, thus the preparation condition of GMA $5.0 \mathrm{~g}$, PVP-K30 $1.0 \mathrm{~g}$, reaction temperature $70^{\circ} \mathrm{C}$, reaction time $12 \mathrm{~h}$, ratio of ethanol to water 87:13 were chosen for the fabrication of FPGMA microsphere.

Based on the preparation of PGMA microspheres, four fluorescent molecules (FITC, RhB, PhB and SR101) were fed into the mixture of monomer, crosslinking reagent and initiator to synthesize the fluorescent FPGMA microspheres. During the polymerization process, the fluorescent molecules were incorporated and fixed stably in the FPGMA microspheres by the inclusion of FPGMA polymer chains and the affinity force including hydrogen-bond interaction, Van der Waals force and other multiple interactions between functional groups (epoxide groups and carbonyl groups) in PGMA and the active groups from the fluorescent molecules including hydroxyl, carbonyl, amino and sulfonic acid groups $[8,13,33]$. Single-fluorescent FPGMA microspheres doped with FITC, RhB, PhB and SR101 dyes were prepared. The fluorescent images and emission spectra of the prepared products are shown in Figure 3. The results showed that the fluorescent molecules have been incorporated into the formed FPGMA microspheres.

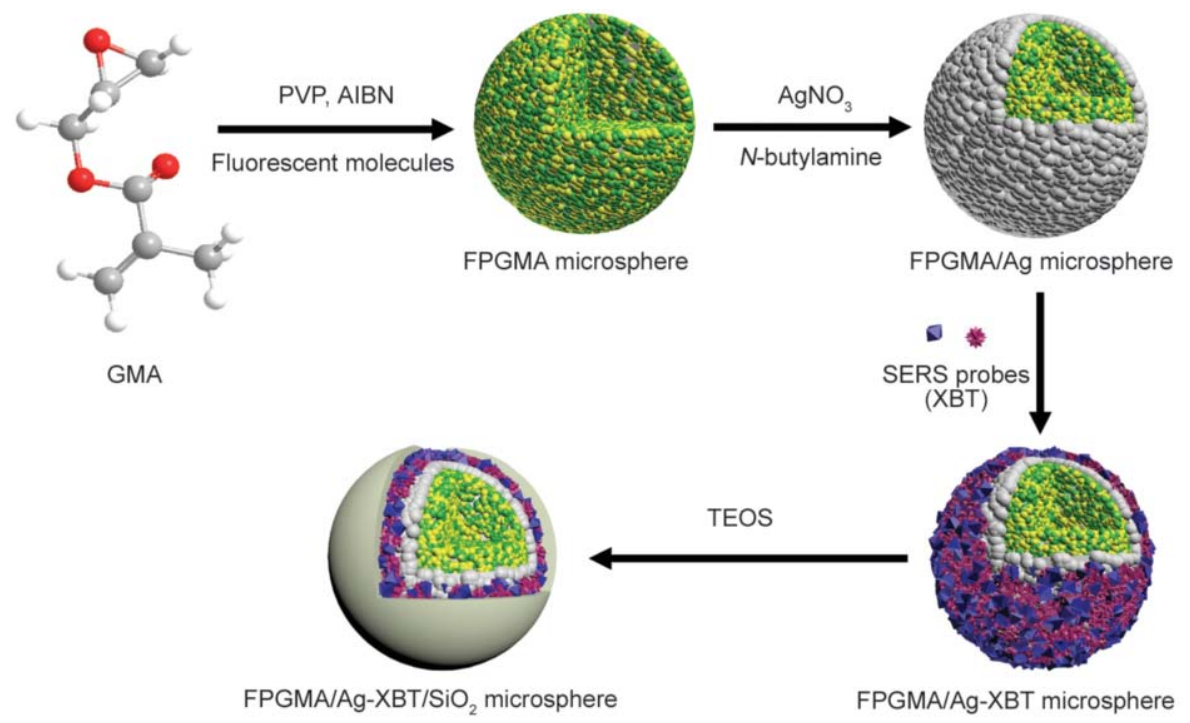

Figure 1. Preparation process of the encoded FPGMA/Ag-XBT/ $\mathrm{SiO}_{2}$ microspheres. 


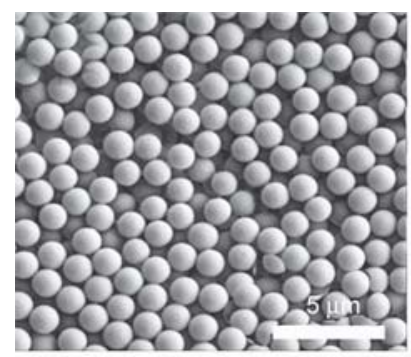

a)

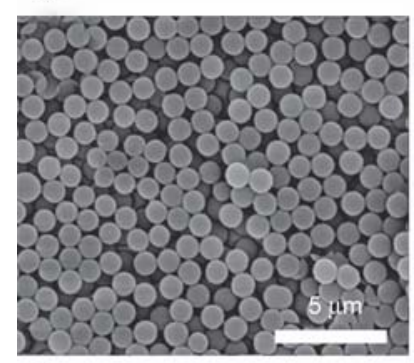

e)

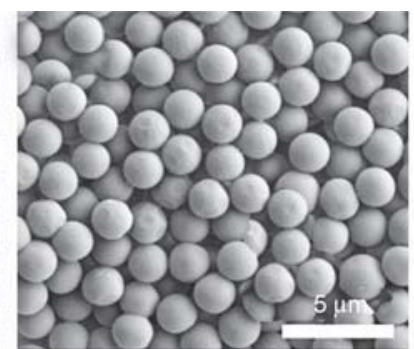

b)

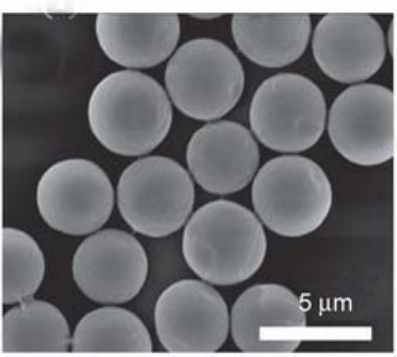

f)

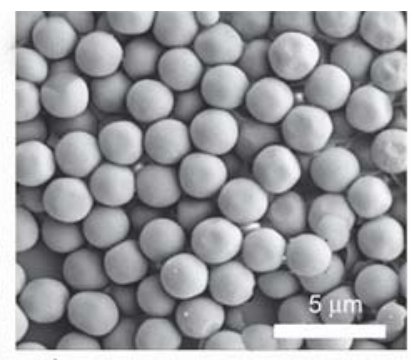

c)

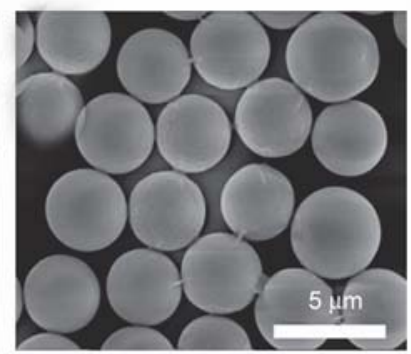

g)

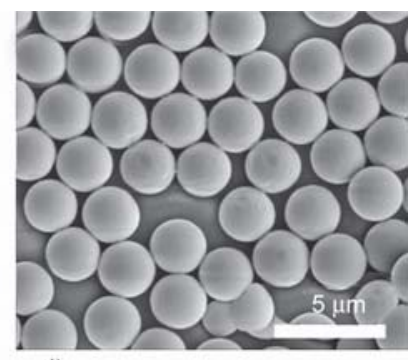

d)

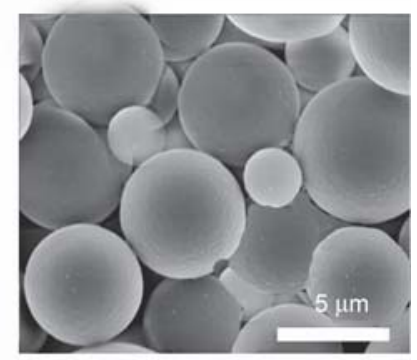

h)

Figure 2. SEM images of the PGMA microspheres synthesized with various dosage of PVP-K30: (a) $3.0 \mathrm{~g}$, (b) $1.5 \mathrm{~g}$, (c) $1.0 \mathrm{~g}$, (d) $0.5 \mathrm{~g}$. SEM imagines of PGMA microspheres with various ratio of ethanol to water: (e) 95:15, (f) 87:13, (g) 86:14, (h) 85:15, GMA 5.0 g, PVP-K30 1.0 g, reaction temperature $70^{\circ} \mathrm{C}$, reaction time $12 \mathrm{~h}$.

Table 1. Average diameters and polydispersity index (PDI) of the PGMA microspheres corresponding to Figure 2.

\begin{tabular}{|l|c|c|c|c|c|c|c|c|}
\hline Serial number & (a) & (b) & (c) & (d) & (e) & (f) & (g) & (h) \\
\hline diameter $[\mu \mathrm{m}]$ & 0.81 & 1.25 & 1.72 & 1.81 & 2.40 & 3.13 & 3.17 & 4.78 \\
\hline PDI $^{*}$ & 0.07 & 0.04 & 0.03 & 0.10 & 0.05 & 0.03 & 0.10 & 0.32 \\
\hline
\end{tabular}

${ }^{*} \mathrm{PDI}=($ Standard deviation of microsphere diameter/average microsphere diameter $)^{2}$.

Observed by the fluorescence microscope, the prepared FPGMA microspheres were uniform in size and presented bright fluorescence. All the FPGMA microspheres exhibited single fluorescence emission (520, 585575 and $608 \mathrm{~nm}$, respectively) under the excitation of $488 \mathrm{~nm}$. Two dyes were simultaneously incorporated into the FPGMA microspheres for multiple fluorescence encoding. As shown in Figure 4, FITC and $\mathrm{RhB}$ with different ratio were incorporated in the FPGMA microspheres. The fluorescence emission spectra of double-fluorescent microspheres (Figure 5) showed two characteristic emissions (520 and $586 \mathrm{~nm}$ ) of FITC and RhB excited by $488 \mathrm{~nm}$, indicating that the two fluorescent dyes were doped simultaneously into the microspheres without spectra overlap. According to Figure 4 and Figure 5, it was found that the color and emission properties changed with the change of dosage ratio of FITC to $\mathrm{RhB}$ (range in $8: 1$ to $1: 2$ ). The intensity of the emission peaks can be tuned by adjusting the feeding ratio of
FITC and RhB, which implied the microsphere could be encoded with different fluorescence intensity.

\subsection{Preparation and characterization of PGMA/Ag microspheres}

The Ag nanoparticles were deposited on the surface of PGMA microspheres through the reduction of $\mathrm{AgNO}_{3}$ in the presence of butylamine. The mass ratios of $\mathrm{AgNO}_{3}$ to PGMA microspheres were varied from $0: 1$ to $16: 1$. The SEM images of PGMA/Ag microspheres prepared at different mass ratios of $\mathrm{AgNO}_{3}$ to PGMA microspheres were shown in Figure 6. When the ratio was over 2:1 (Figure 6c), the surface of the microspheres became rough. When the feeding ratio was 4:1 (Figure 6d), the Ag nanoparticles covered the whole microspheres, and with the feeding ratio increased to 16:1 (Figure 6f), it could be observed a large number of Ag particles were deposited on the surface of the microspheres. The PGMA/Ag microspheres prepared with 8:1 mass ratio of $\mathrm{AgNO}_{3}$ to PGMA was characterized by thermogravimetric analysis (Figure 7a) and the X-ray diffraction pattern (Figure $7 b$ ) for the further investigation of material properties.

The thermogravimetric analysis and the X-ray diffraction pattern in Figure 7 further confirmed the deposition of Ag nanoparticles on the surface of the microspheres. Several mass loss steps were observed in Figure 7a, a mass loss step is observed 
a)

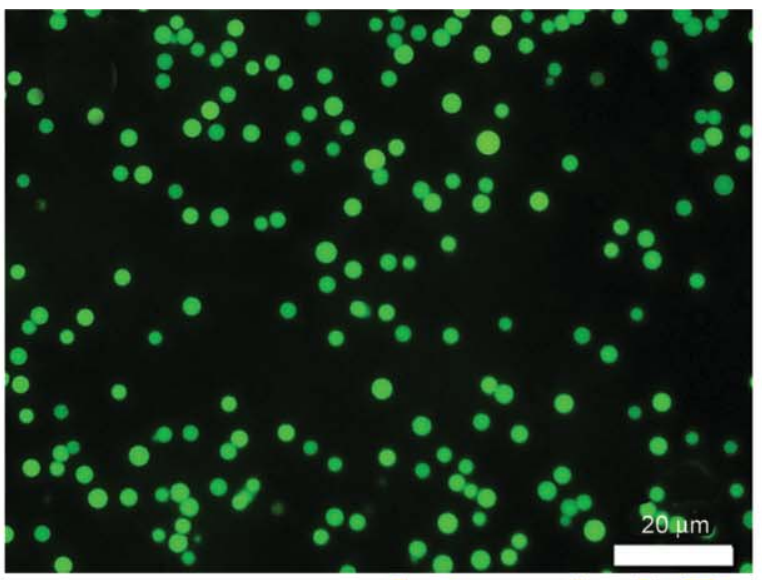

b)
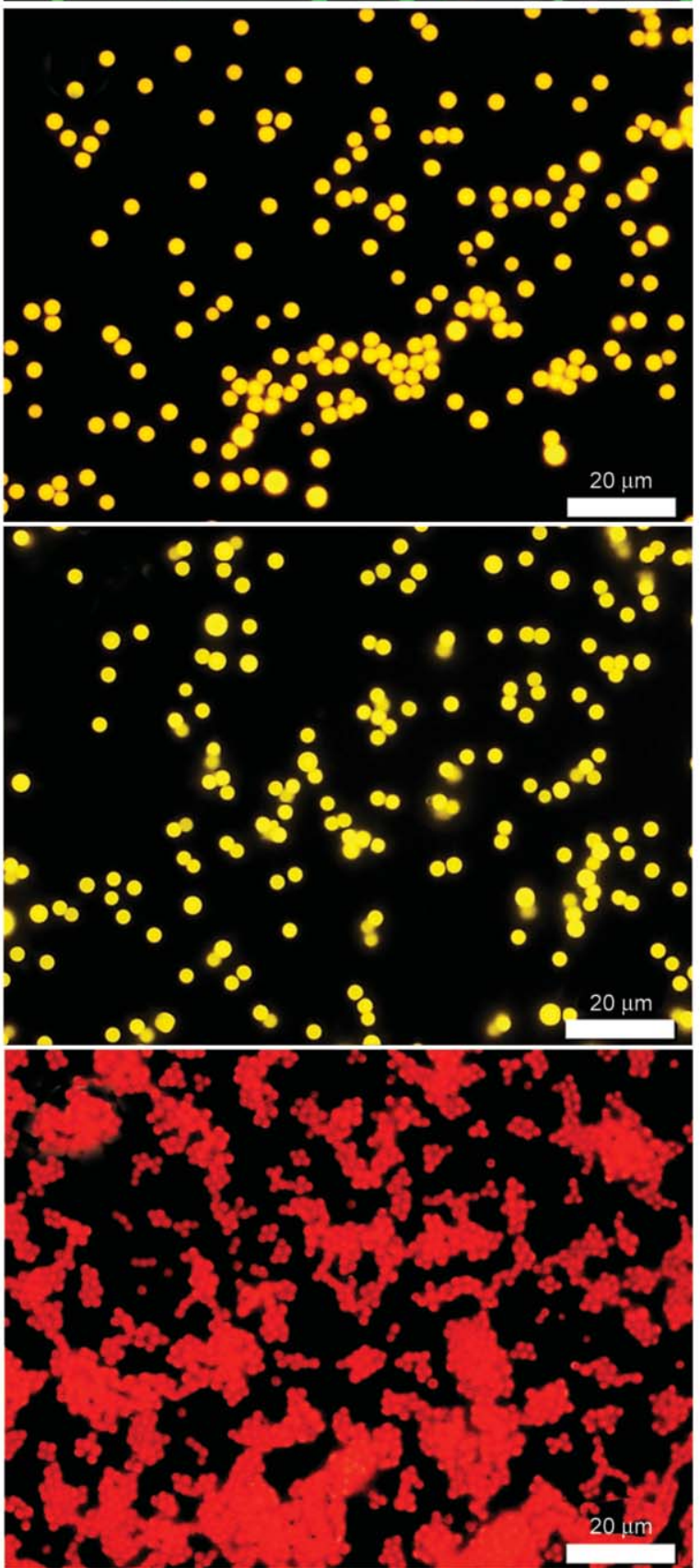
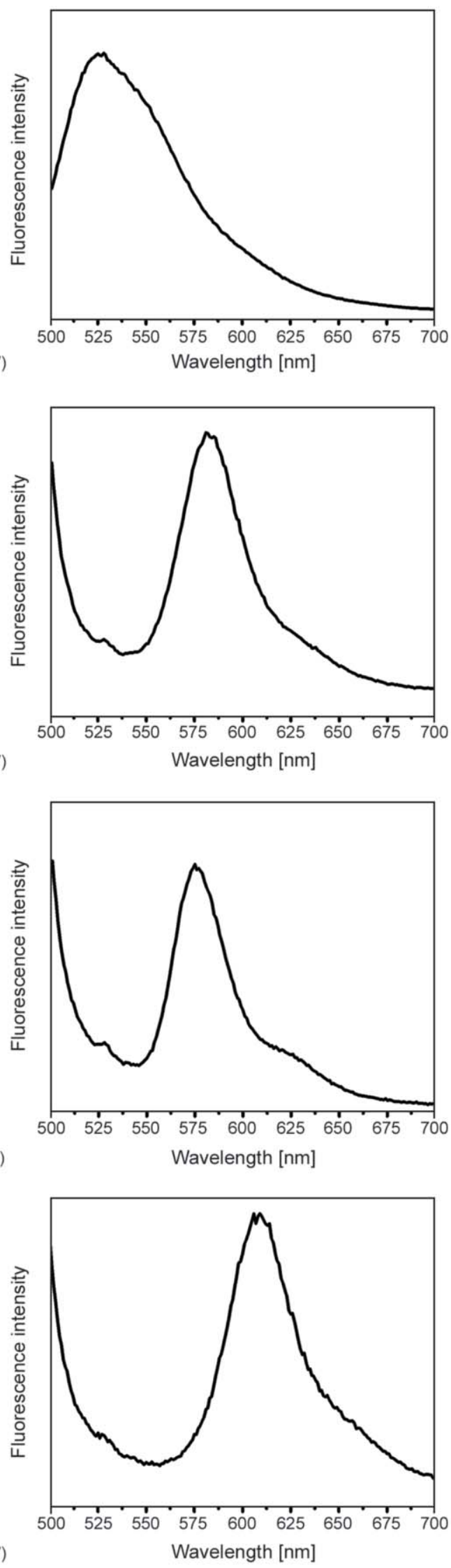

Figure 3. Fluorescence images of FPGMA microspheres encoded with (a) FITC, (b) RhB, (c) PhB, (d) SR101; the fluorescence spectra of FPGMA microspheres encoded with (a') FITC, (b') RhB, (c') PhB, (d') SR101, with an excitation of $488 \mathrm{~nm}$. 

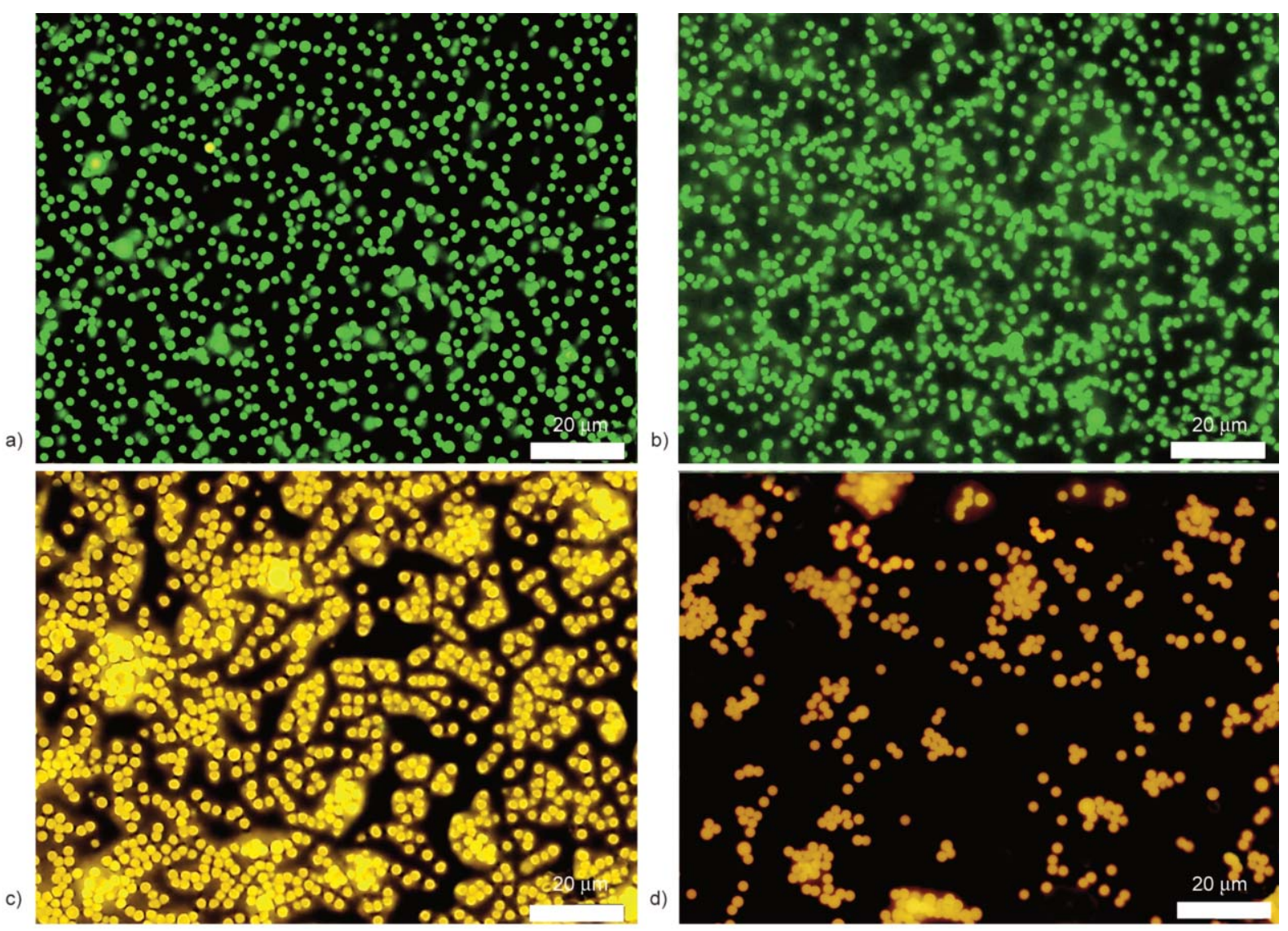

Figure 4. The fluorescence images of FPGMA microspheres encoded by different dosage ratio: of FITC to RhB (a) 8:1, (b) $4: 1$, (c) $1: 1$, (d) $1: 2$.

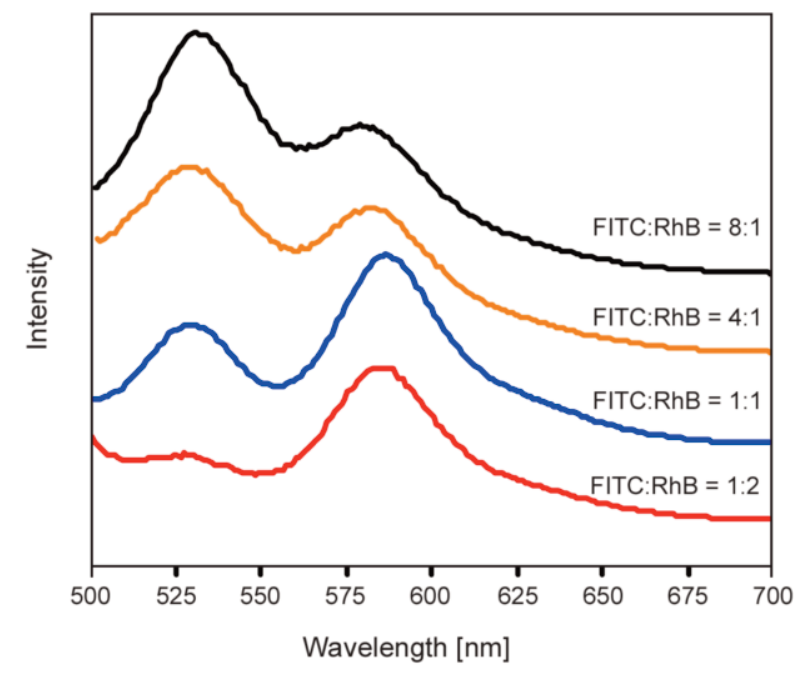

Figure 5. Emission spectra of FPGMA microspheres encoded by FITC and RhB with different dosage ratio. The excitation wavelength was $488 \mathrm{~nm}$.

from room temperature to $200^{\circ} \mathrm{C}$ and is attributed to the dehydration of the microspheres. A second mass loss step is observed over the 200 to $400{ }^{\circ} \mathrm{C}$ temperature range and is due to the oxidation and carbonation of PGMA resin. For PGMA microspheres and PGMA/Ag microspheres, the total weight losses are 98 and $90 \%$ respectively, and it indicated that the PGMA/Ag microspheres contain Ag nanoparticles. The amount of Ag deposited on the PGMA microspheres was about $8.0 \%$ in weight. The XRD pattern of PGMA/Ag microspheres is presented in Figure $7 \mathrm{~b}$. In the $2 \theta$ range of $20-40^{\circ}$, a broad peak belong to the amorphous structure of the PGMA microspheres. Three characteristic peaks for Ag were observed at $2 \theta$ angles of $44.63,51.88,76.65^{\circ}$ and the peak position could be indexed to (200), (220), and (311) crystalline planes of the structure of $\mathrm{Ag}$ (JCPDS No.04-0783) [33, 34].

The surface enhanced Raman spectral (SERS) activity of PGMA/Ag microspheres fabricated with different mass ratios of $\mathrm{AgNO}_{3}$ to PGMA were tested using 4-CBT as tag molecule. The SERS spectrum of 4-CBT by PGMA/Ag microspheres with different mass ratio of $\mathrm{AgNO}_{3}$ to PGMA microspheres was shown in Figure 8. It was found that the intensity of the characteristic SERS peaks is enhanced obviously when the mass ratios of $\mathrm{AgNO}_{3}$ to PGMA increased from 0:1 to 8:1, suggesting the PGMA/Ag possessed excellent SERS activity [30]. When the amount of $\mathrm{AgNO}_{3}$ increased, the $\mathrm{Ag}$ nanoparticles deposited on 


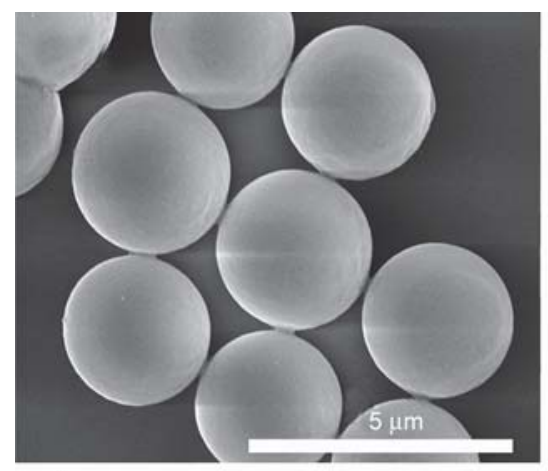

a)

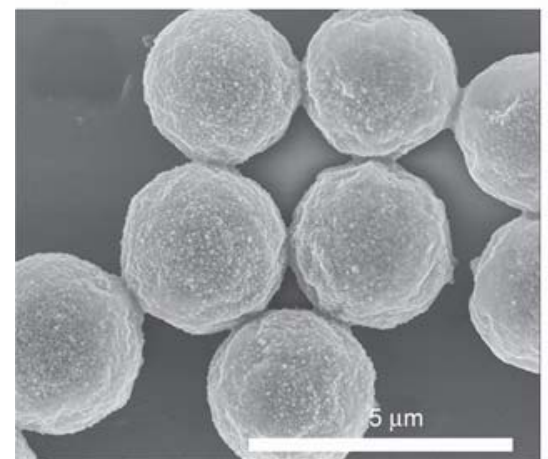

d)

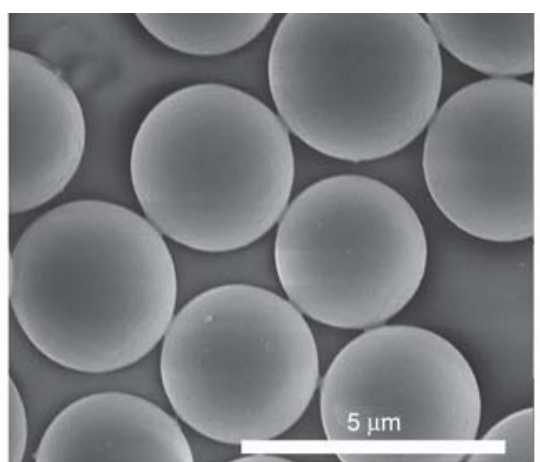

b)

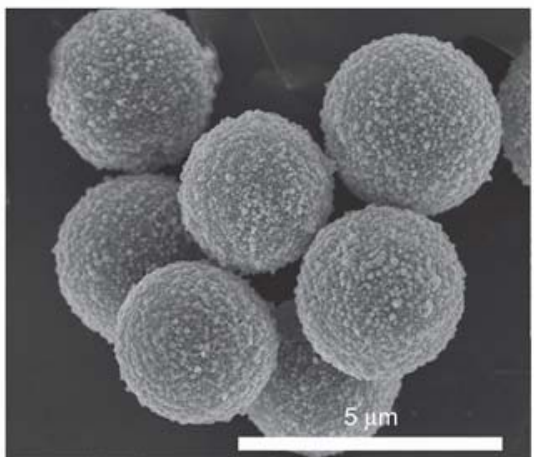

e)

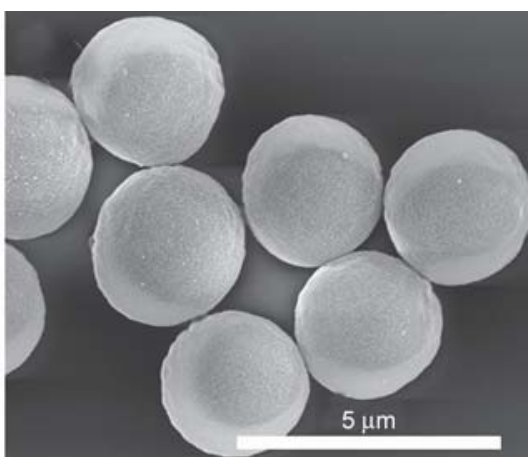

c)

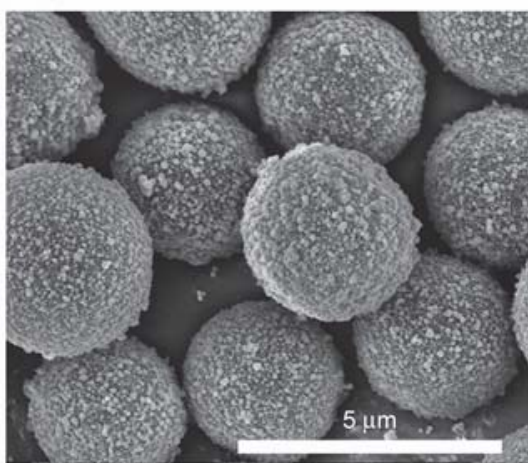

f)

Figure 6. SEM images of PGMA/Ag microspheres with different mass ratio of $\mathrm{AgNO}_{3}$ to PGMA microspheres: (a) 0:1, (b) $1: 1$, (c) $2: 1$, (d) $4: 1$, (e) $8: 1$, (f) $16: 1$.

PGMA surface increased and the hot spots increased, thus the intensity of the characteristic SERS peaks was enhanced [27, 28]. Moreover, when Ag nanoparticles deposited on the PGMA increased the gap sites between two Ag nanoparticles became closer and result in SERS enhancement. As it is reported, closely spaced interacting particles can provide extra surface plasmon enhancement, particularly near the gap sites between two particles in proximity [35-37]. The SERS intensity activity change slightly when the mass ratio is over $8: 1$. This indicated that the surface

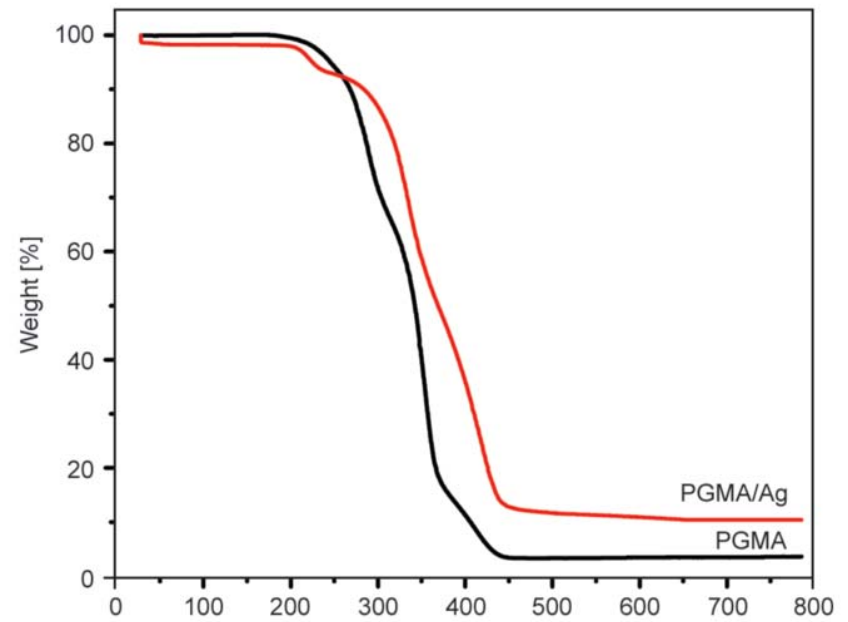

a)

Temperature $\left[{ }^{\circ} \mathrm{C}\right]$ enhanced Raman signal is saturated when the mass ratio is over 8:1, which was used as SERS encoding for the further experiments.

\subsection{SERS encoding of PGMA/Ag microspheres}

PGMA/Ag microspheres (prepared with a mass ratio of $\mathrm{AgNO}_{3}$ to PGMA was 8: 1) was encoded by single SERS reporters 4-ABT, 4-CBT, 4-HBT and double SERS reporters ABT-HBT, ABT-CBT, CBT-HBT respectively. The SERS spectra of the microspheres

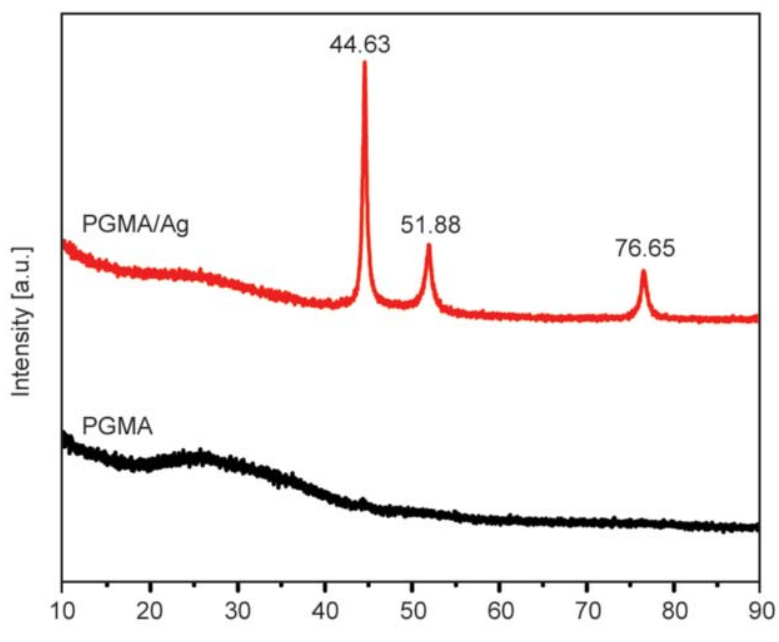

b)

Figure 7. (a) The thermogravimetric analysis of PGMA/Ag microspheres; (b) the XRD pattern of PGMA/Ag microspheres. 


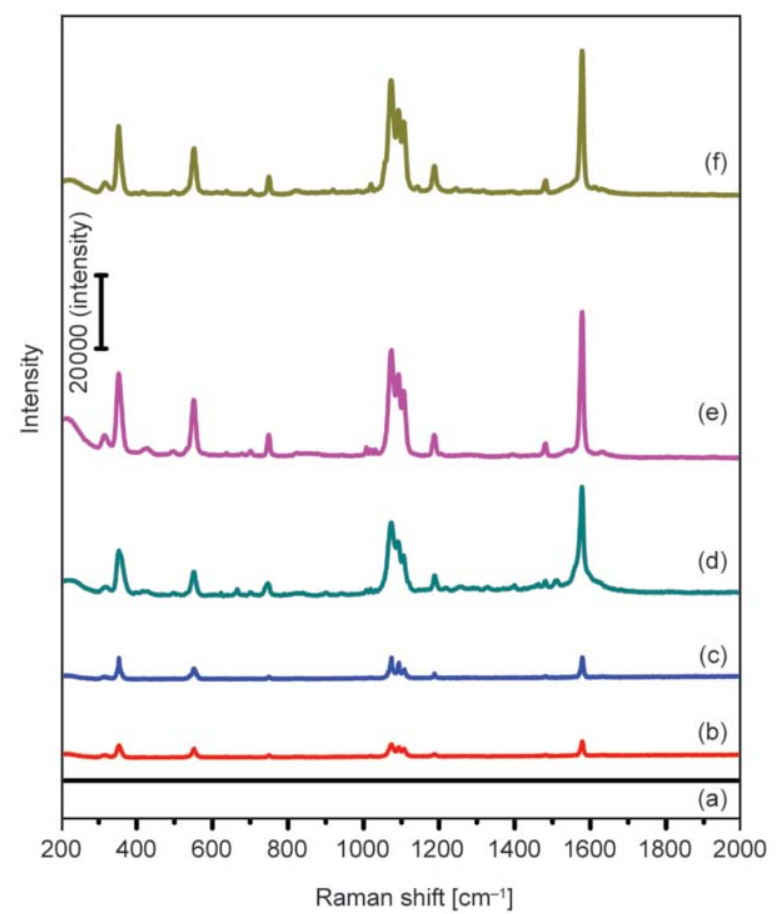

Figure 8. Raman spectrum of PGMA/Ag microspheres with different mass ratio of $\mathrm{AgNO}_{3}$ to PGMA microspheres immobilized with 4-CBT: (a) 0:1, (b) 1:1, (c) $2: 1$, (d) $4: 1$, (e) $8: 1$, (f) $16: 1$.

encoded by 4-ABT, 4-CBT, 4-HBT, ABT-HBT (1:1, by weight), ABT-HBT (1:2), ABT-CBT (1:1), CBTHBT $(1: 1)$ were shown in Figure 9. The SERS encoding signal of 4-CBT was characterized by peak at $350,554,1580 \mathrm{~cm}^{-1}$ and three continuous peaks between 1066 and $1159 \mathrm{~cm}^{-1}$. The SERS characteristic peaks of ABT were at 1074, 1144, 1197, 1390, 1436 and $1580 \mathrm{~cm}^{-1}$. While the characteristic encoding

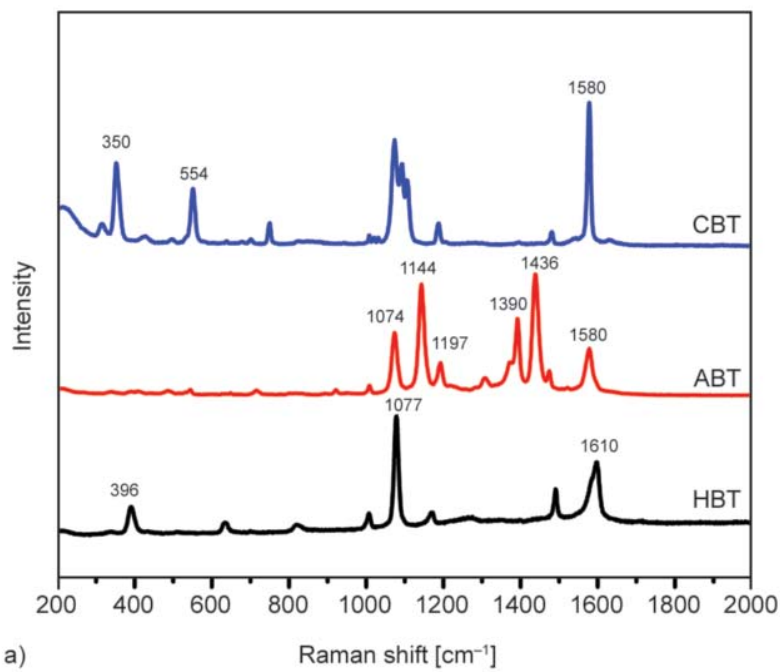

signals of 4-HBT were at 396, 1077 and $1610 \mathrm{~cm}^{-1}$. Figure 9b showed the SERS coding signal of twoSERS probes encoded PGMA/Ag microspheres can be easily identified. For the encoding of ABT-HBT (1:1), not only the characteristic peaks of 4-ABT can be observed, but also the three characteristic peaks of 4-HBT at 396, 1077, $1610 \mathrm{~cm}^{-1}$ were easily distinguished. For CBT-HBT (1:1) encoding, the characteristic peaks at $350,554,1583 \mathrm{~cm}^{-1}$ and a group of peaks at 1066 1159 $\mathrm{cm}^{-1}$ were originated from 4CBT, while the peaks at $396,1077,1610 \mathrm{~cm}^{-1}$ were originated from 4-HBT. When ABT-CBT (1:1) was used as SERS coding, the peaks of 4-ABT and 4CBT at 1580,1436, $1144 \mathrm{~cm}^{-1}$ and 350, 554, 1066 $1120 \mathrm{~cm}^{-1}$ can be observed respectively. The multiple SERS encoding could be explained by favorable synergy of strong electromagnetic enhancement and chemical enhancement caused by the coated Ag nanoparticles $[35,36]$. The above results showed that different SERS reporters can be used for SERS encoding of PGMA/Ag microspheres simultaneously, and the characteristic coding signals of multiple SERS reporters can be clearly distinguished without interfere with each other.

\subsection{Fluorescence and SERS joint encoded FPGMA/Ag-XBT/ $\mathrm{SiO}_{2}$ microspheres}

In order to increase the encoding capacity of the microspheres, the as prepared fluorescent FPGMA microspheres were deposited with Ag nanoparticles on the surface (FPGMA/Ag) and modified by SERS reporters for fluorescence and SERS joint encoding.

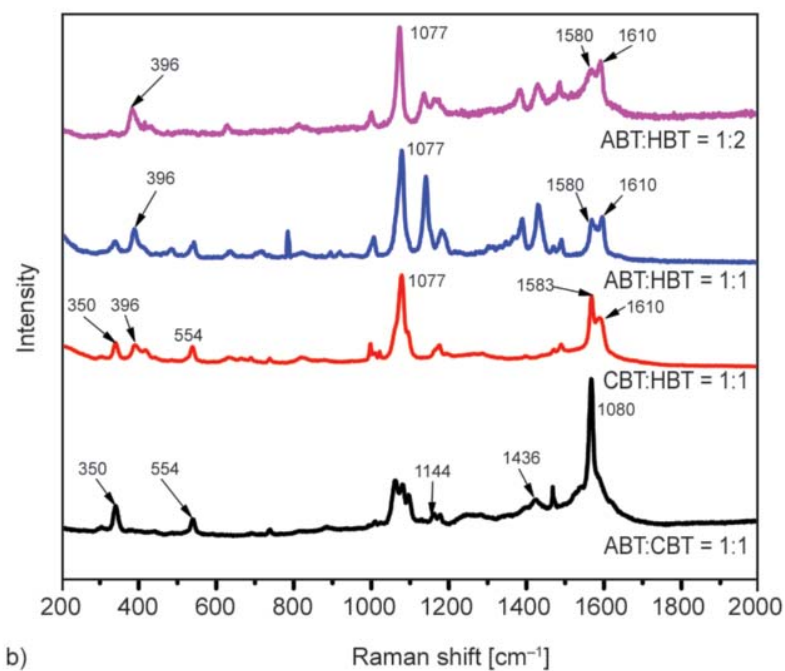

Figure 9. SERS encoding signals of PGMA/Ag microspheres immobilized with (a) 4-ABT, 4-CBT and 4-HBT as single SERS reporters, (b) ABT-HBT (1:2), ABT-HBT (1:1), CBT-HBT (1:1) and ABT-CBT (1:1) as double SERS reporters. 
Then the as fabricated encoding microspheres were fication for the potential applications in fields of bicoated with $\mathrm{SiO}_{2}$, which would beneficial for modi- ological detection and biological probes.

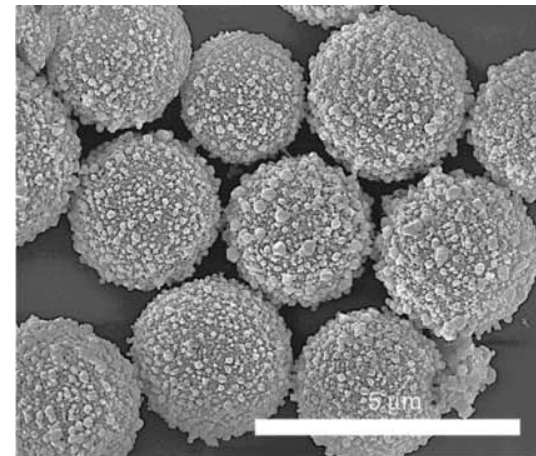

a1)

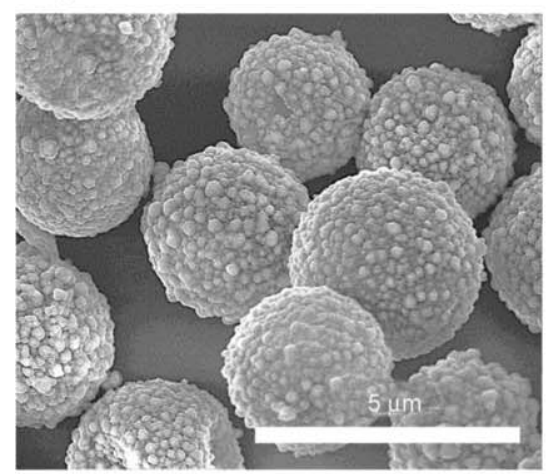

a2)

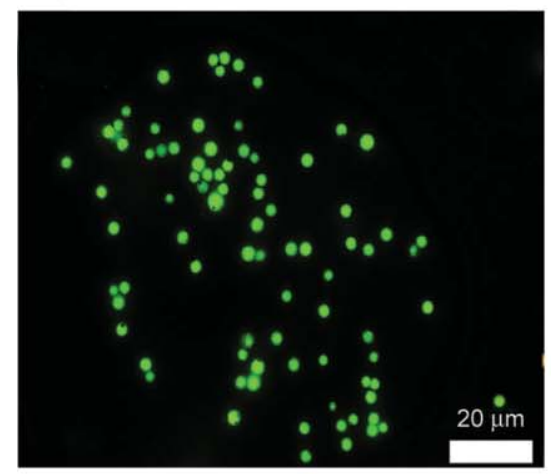

a3)

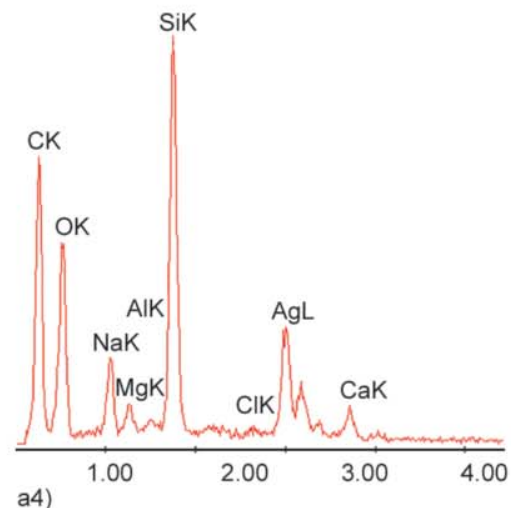

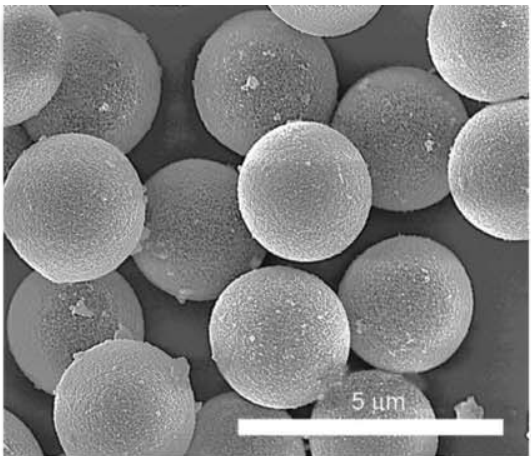

b1)

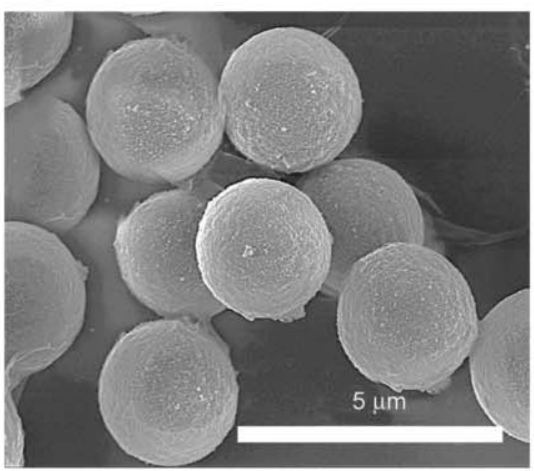

b2)

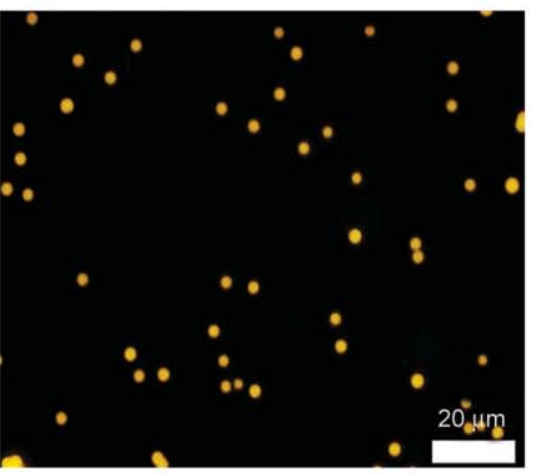

b3)

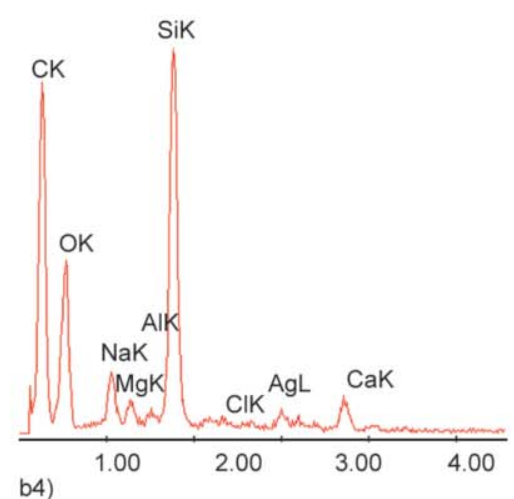

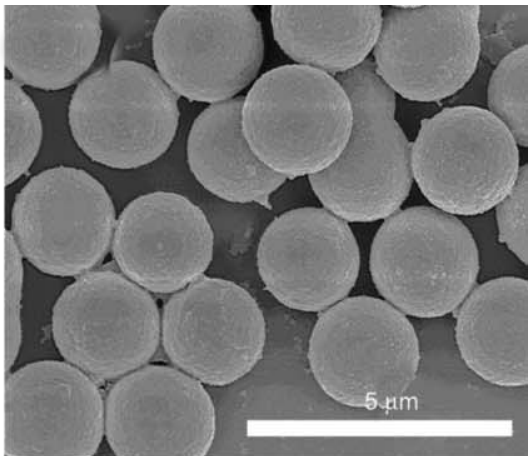

c1)

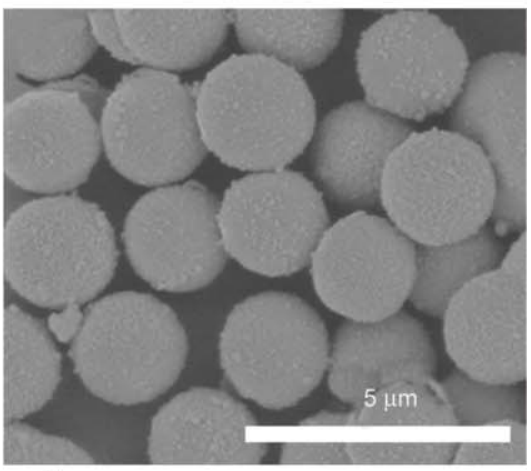

c2)

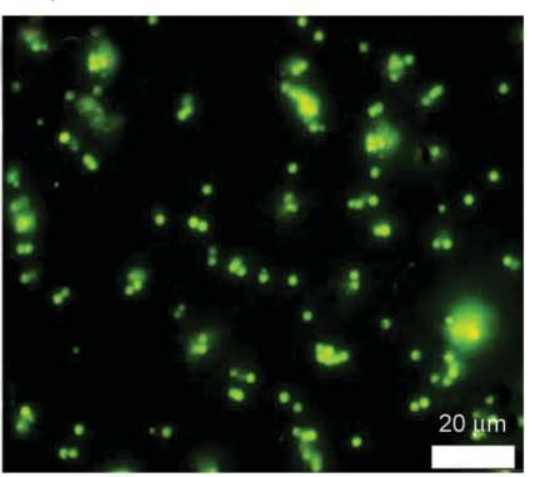

c3)

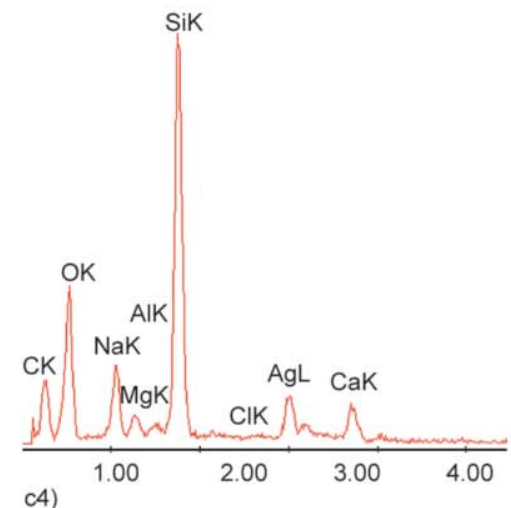

Figure 10. The SEM images of (a1) PGMA-FITC/Ag-ABT, (b1) PGMA-RhB/Ag-HBT, (c1) PGMA-(FITC-RhB)/Ag-(ABTHBT), (a2) PGMA-FITC/Ag-ABT/SiO 2 , (b2) PGMA-RhB/Ag-HBT/SiO 2 and (c2) PGMA-(FITC-RhB)/Ag(ABT-HBT)/SiO ${ }_{2}$. The fluorescence images of (a3) PGMA-FITC/Ag-ABT/SiO 2 , (b3) PGMA-RhB/Ag-HBT/SiO ${ }_{2}$ and (c3) PGMA-(FITC-RhB)/Ag-(ABT-HBT)/SiO ${ }_{2}$. The EDX spectrum of (a4) PGMA-FITC/Ag-ABT/SiO $\mathrm{S}_{2}$, (b4) PGMA-RhB/Ag-HBT/SiO 2 and (c4) PGMA-(FITC-RhB)/Ag-(ABT-HBT)/SiO 2 . 
Three sets of fluorescence-SERS joint encoded microspheres including PGMA-FITC/Ag-ABT/SiO GMA-RhB/Ag-HBT/SiO ${ }_{2}$ and PGMA-(FITC-RhB)/ $\mathrm{Ag} / \mathrm{SiO}_{2}$ were prepared. The SEM and fluorescence images of the dual model encoded microspheres were shown in Figure 10. Compared to the smooth surface of PGMA microspheres, the surface of PGMA-FITC/ Ag-ABT, PGMA-RhB/Ag-HBT and PGMA-(FITC$\mathrm{RhB}) / \mathrm{Ag}$ microspheres were very rough (see Figure 10a1-10c1), which indicated that Ag nanoparticles were coated on the surface of the microspheres. In Figure 10a2-10c2, the rough Ag nanoparticles surface disappeared and a smooth shell was coated on the surface of PGMA-FITC/Ag-ABT/SiO 2 , PGMA$\mathrm{RhB} / \mathrm{Ag}-\mathrm{HBT} / \mathrm{SiO}_{2}$ and PGMA-(FITC-RhB)/Ag/ $\mathrm{SiO}_{2}$. It indicated a silica shell was coated on the microspheres. The samples were also tested by characterized by EDX spectrum and the results showed the

a)
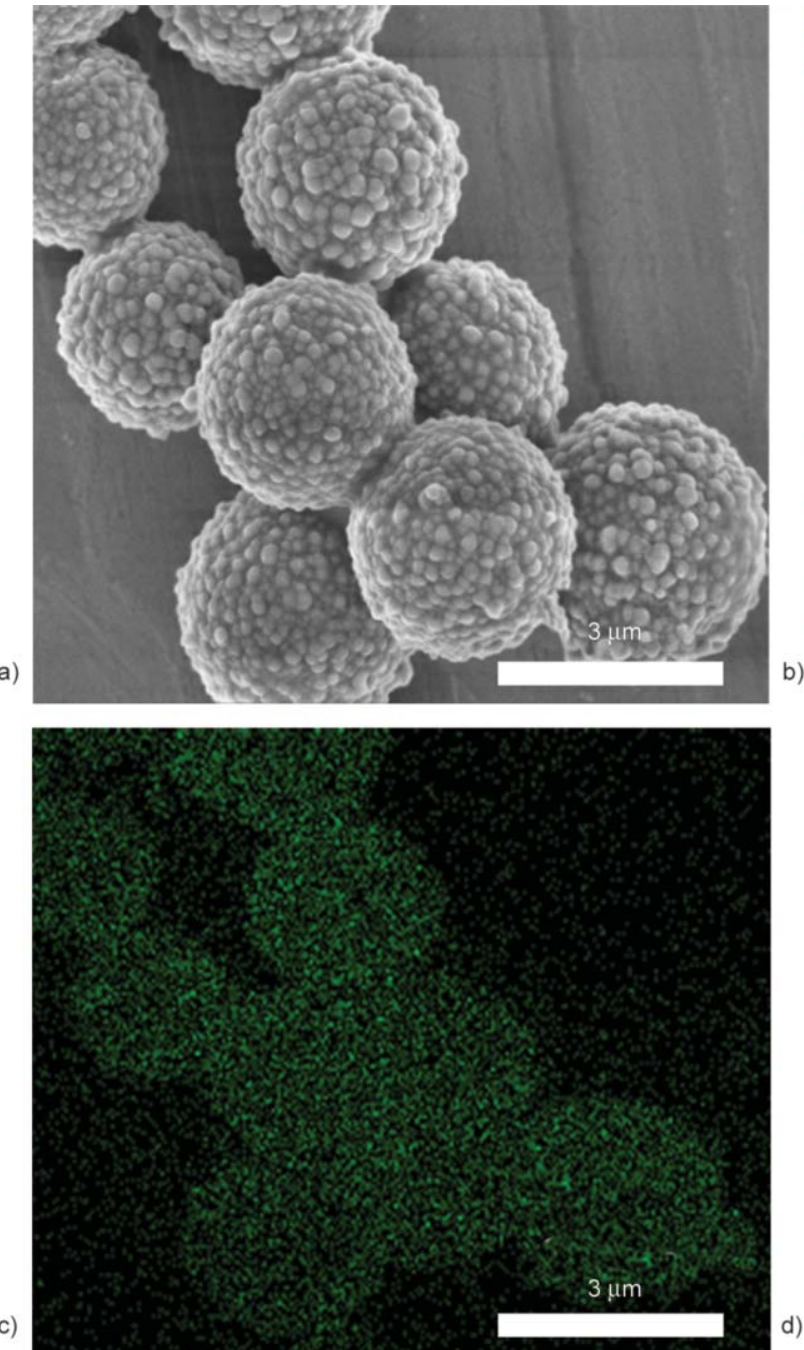

presence of $\mathrm{Ag}$ and $\mathrm{Si}$ (Figure 10a4-10c4), confirming the successful $\mathrm{Ag}$ nanoparticle deposition and $\mathrm{SiO}_{2}$ coating. The dual model encoded PGMAFITC/ Ag-ABT/ $/ \mathrm{SiO}_{2}$ microspheres were characterized by TEM (Figure 11a) and elemental mapping as an example to investigate the surface distribution of $\mathrm{Ag}$ nanoparticles and $\mathrm{SiO}_{2}$. The elemental mapping images showed the uniform distribution of Ag (Figure 11c) and Si (Figure 11b) elements in the PGMAFITC/Ag-ABT/SiO ${ }_{2}$ microspheres and the thickness of the silicon dioxide layer is about $30 \mathrm{~nm}$ from the TEM image in Figure 11d. The as prepared three fluorescence-SERS joint encoded microspheres were observed by fluorescence microscope and showed green, yellow and yellowish-green fluorescence, respectively (Figure 10a3-10c3).

The characteristic fluorescence and SRES coding signals of these encoded microspheres were detected
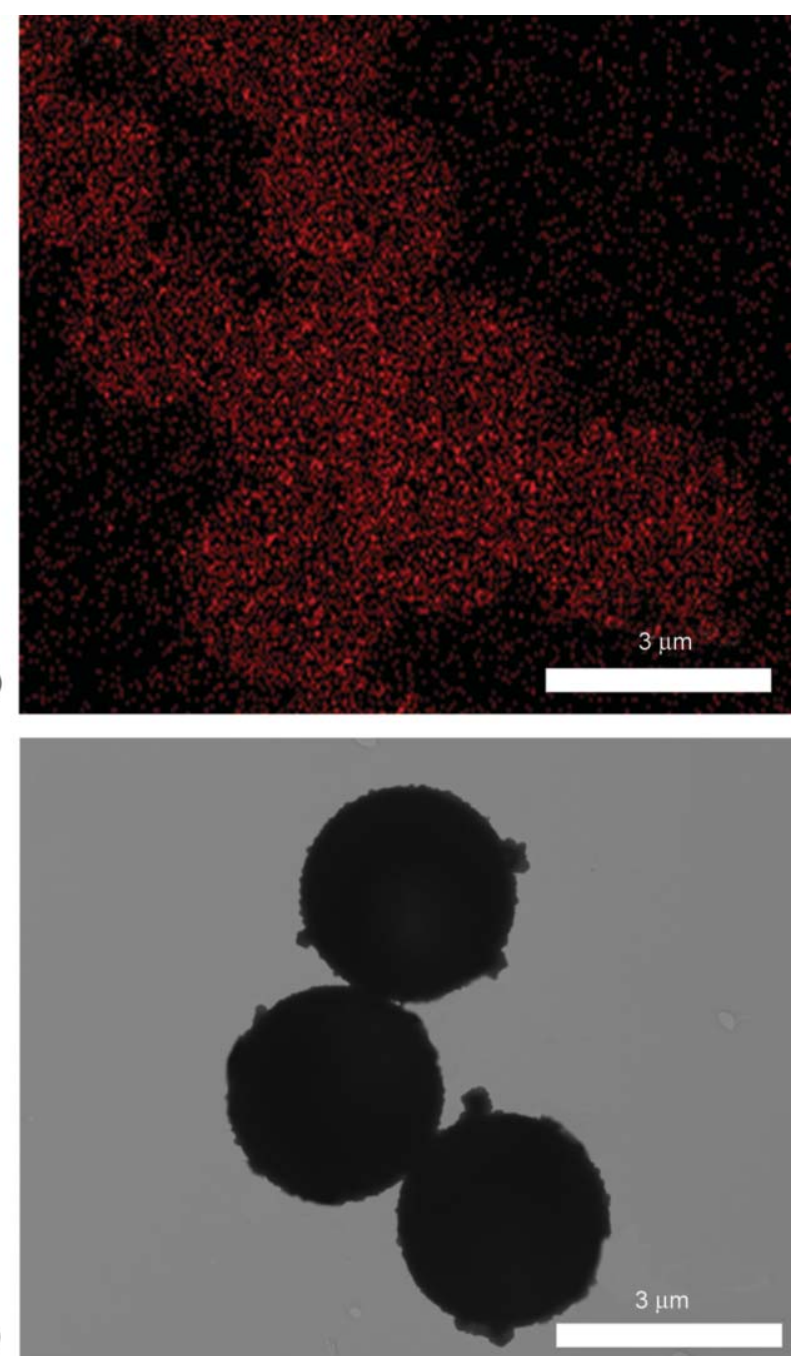

Figure 11. (a) SEM images of PGMA-FITC/Ag-ABT/SiO 2 composite microspheres; (b) Si elemental mapping images of PGMA-FITC/Ag-ABT/SiO 2 microspheres; (c) Ag elemental mapping images of PGMA-FITC/Ag-ABT/SiO 2 microspheres; (d) the TEM images of PGMA-FITC/Ag-ABT/SiO ${ }_{2}$ microspheres. 
(Figure 12). As the combination of FITC and 4-ABT encoding, the prepared FITC-PGMA/Ag-ABT/SiO
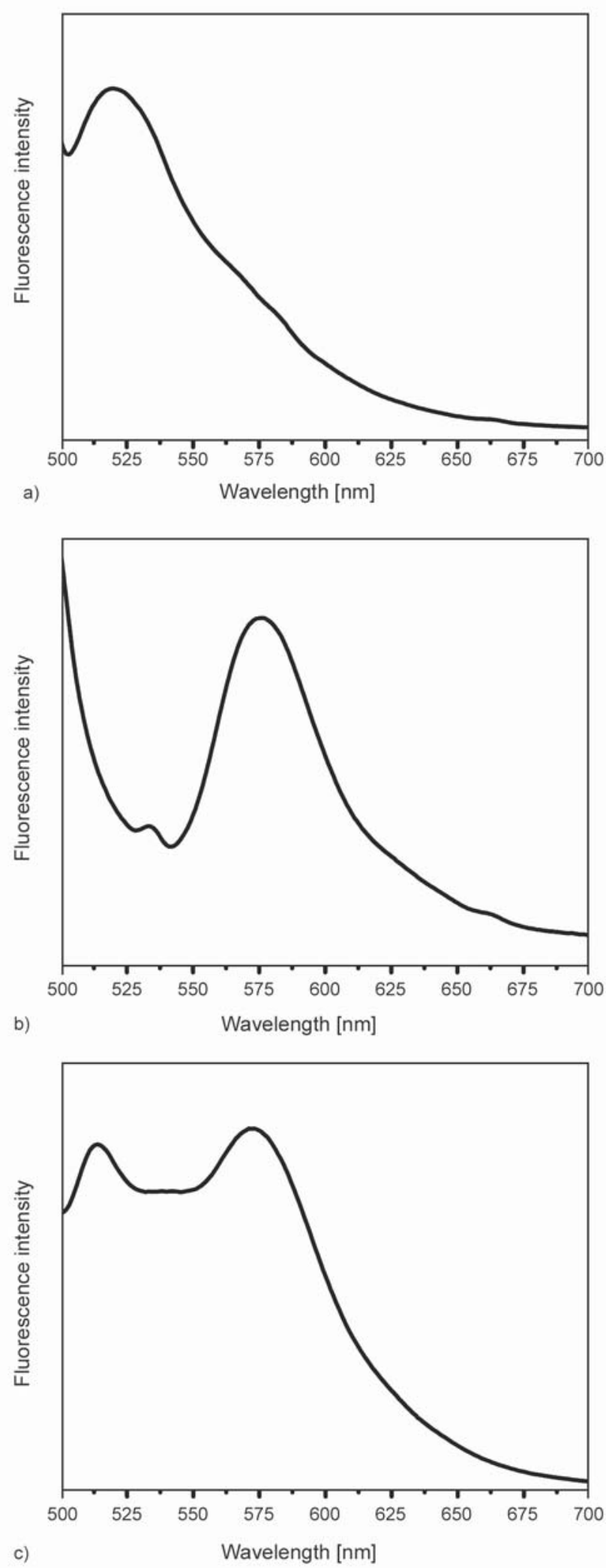

encoded microsphere not only showed the characteristic fluorescence emission of FITC at $520 \mathrm{~nm}$,
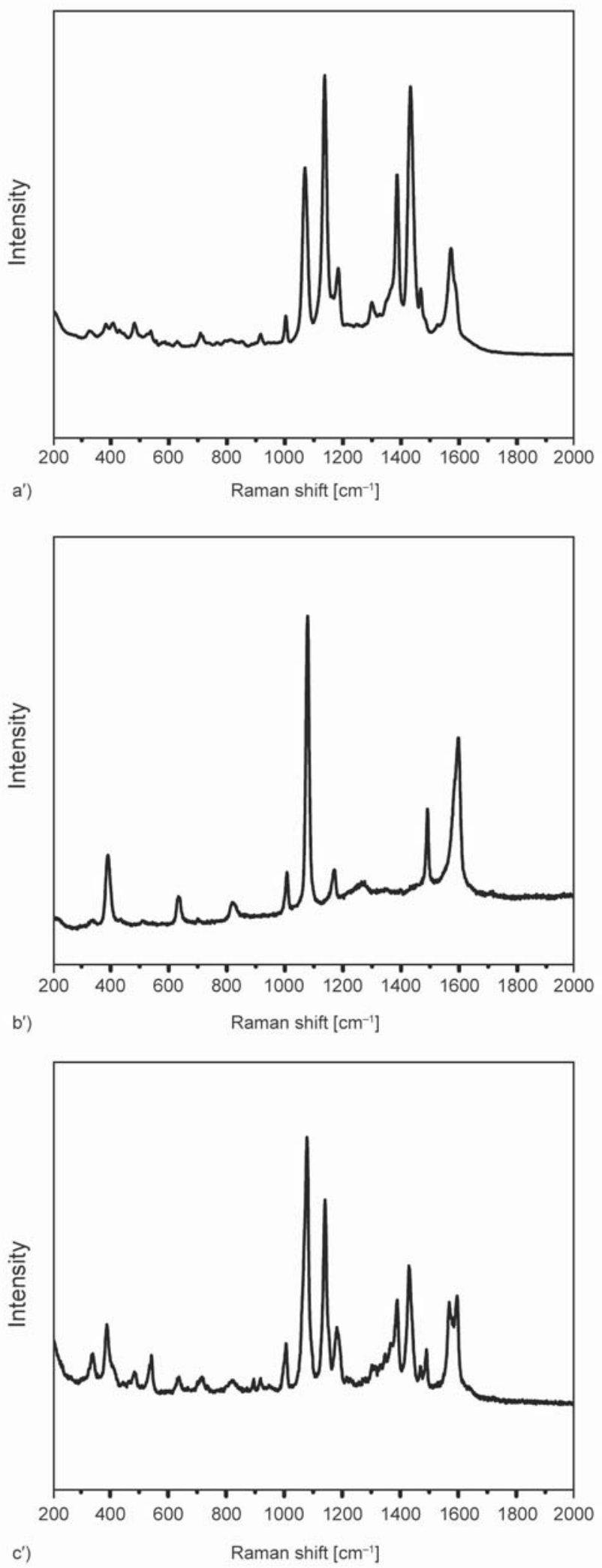

Figure 12. The fluorescence spectra of (a) PGMA-FITC/Ag-ABT/SiO 2 , (b) PGMA-RhB/Ag-HBT/SiO 2 , (c) PGMA-(FITC$\mathrm{RhB}) / \mathrm{Ag}-(\mathrm{ABT}-\mathrm{HBT}) / \mathrm{SiO}_{2}$, the Raman spectrum of (a') PGMA-FITC/Ag-ABT/SiO ${ }_{2},\left(\mathrm{~b}^{\prime}\right) \mathrm{PGMA}-\mathrm{RhB} / \mathrm{Ag}-$ $\mathrm{HBT} / \mathrm{SiO}_{2},\left(\mathrm{c}^{\prime}\right)$ PGMA-(FITC-RhB)/Ag-(ABT-HBT)/SiO 2 . The excitation wavelength was $488 \mathrm{~nm}$. 
but also the characteristic SERS signals of $\mathrm{ABT}$ at 1074, 1144, 1197, 1390, 1436 and $1580 \mathrm{~cm}^{-1}$ (Figure 12a and 12a'). As it shown in Figure 12b and 12b', the RhB-PGMA/Ag-HBT/ $/ \mathrm{SiO}_{2}$ presented the distinct fluorescence emission $(583 \mathrm{~nm})$ of $\mathrm{RhB}$ and the characteristic SERS peaks at 396, 1077, $1610 \mathrm{~cm}^{-1}$ derived from 4-HBT. For the joint multiple encoding of two fluorescent probes (FITC-RhB) and two SERS reporters (ABT-HBT), the characterized fluorescence emissions and SERS spectra can be clearly identified and easily distinguished (Figure $12 \mathrm{c}$ and 12c'). The PGMA-(FITC-RhB)/Ag-(ABT-HBT)/ $\mathrm{SiO}_{2}$ microspheres displayed the characteristic fluorescence emissions of FITC $(520 \mathrm{~nm})$ and $\mathrm{RhB}$ $(583 \mathrm{~nm})$, and the characteristic SERS spectra of 4ABT $\left(1580,1436\right.$ and $\left.1144 \mathrm{~cm}^{-1}\right)$ and 4-HBT (396, 1077 and $\left.1610 \mathrm{~cm}^{-1}\right)$. The results indicated the combination of fluorescence and SERS successfully expands the encoding capacity of the microspheres. Due to the fluorescence and SERS spectra in two optical channels, the encoding approach avoided spectral overlaps and was beneficial for a large encoding capacity of microspheres $[10,26]$. Adopting this method, many other fluorescence probes and SERS reporters also can be used in SERS-fluorescence combined system with distinctive fluorescence and SERS signals. Therefore, the number of codes can be increased greatly by increasing the number of different fluorescence probes and SERS reporters.

\section{Conclusions}

The present work has designed and prepared a series of new fluorescence and SERS encoded microspheres based on PGMA microspheres. The PGMA were fabricated by dispersion polymerization and encoded by four fluorescence molecules (FITC, RhB, $\mathrm{PhB}$ and SR101) and three SERS probes (4-ABT, 4$\mathrm{CBT}, 4-\mathrm{HBT})$. The as prepared microspheres were uniform in size. Single-fluorescent FPGMA microspheres doped with FITC, RhB, PhB and SR101 dyes were prepared. Multiple dyes could be simultaneously incorporated into the PGMA microspheres and the intensity of the fluorescence can be tuned by adjusting the feeding ratio. PGMA/Ag microspheres were encoded by single SERS reporters 4-ABT, 4-CBT, 4-HBT and double SERS reporters ABT-HBT, ABTCBT, CBT-HBT respectively. Fluorescence and SERS joint encoded FPGMA/Ag-XBT/SiO ${ }_{2}$ microspheres were fabricated and increased the encoding capacity of the microspheres. Three sets of fluorescence-SERS joint encoded microspheres including PGMA-FITC/ Ag-ABT/SiO 2, GMA-RhB/Ag-HBT/SiO 2 and PGMA(FITC-RhB) $/ \mathrm{Ag} / \mathrm{SiO}_{2}$ were prepared. All the fluorescence encoding signals can be excited and detected at $488 \mathrm{~nm}$. Moreover, the encoded microspheres have strong fluorescent emissions and SERS coding signals, and non-overlapping of emission spectra was observed in these encoded microspheres, which greatly increased the encoding capacity. The as prepared fluorescence and SERS encoded microspheres possessed stable spectral encoded signal, large encoded capacity.

\section{Acknowledgements}

We gratefully acknowledge the financial support of the National Natural Science Foundation of China (Grant No. 51503040, 31771893) the Natural Science Foundation of Fujian Province, China (Grant No. 2018J01766) and the Outstanding Youth Research Talent Cultivation Program of Universities in Fujian Province, China (Grant No. 601936).

\section{References}

[1] Leng Y., Wu W., Li L., Lin K., Sun K., Chen X., Li W.: Magnetic/fluorescent barcodes based on cadmium-free near-infrared-emitting quantum dots for multiplexed detection. Advanced Functional Materials, 26, 75817589 (2016).

https://doi.org/10.1002/adfm.201602900

[2] Chen H., Lang Y., Zhang Y., Zhao D., Qin G., Wu C., Zheng K., Qin W.: Dual mode emission of core-shell rare earth nanoparticles for fluorescence encoding. Journal of Materials Chemistry C, 3, 6314-6321 (2015). https://doi.org/10.1039/c5tc00017c

[3] Gong X., Yan H., Yang J., Wu Y., Zhang J., Yao Y., Liu P., Wang H., Hu Z., Chang J.: High-performance fluorescence-encoded magnetic microbeads as microfluidic protein chip supports for AFP detection. Analytica Chimica Acta, 939, 84-92 (2016). https://doi.org/10.1016/j.aca.2016.08.031

[4] Leng Y., Sun K., Chen X., Li W.: Suspension arrays based on nanoparticle-encoded microspheres for highthroughput multiplexed detection. Chemical Society Reviews, 44, 5552-5595 (2015). https://doi.org/10.1039/c4cs00382a

[5] You L., Wang A., Song L., Huang C., Zhang Q.: Synthesis of high quality dual-mode encoded phenolic resin microspheres based on fluorescence and surface enhanced Raman spectra. Materials Letters, 212, 65-68 (2018).

https://doi.org/10.1016/j.matlet.2017.10.069 
[6] Gerver R. E., Gómez-Sjöberg R., Baxter B. C., Thorn K. S., Fordyce P. M., Diaz-Botia C. A., Helms B. A., DeRisi J. L.: Programmable microfluidic synthesis of spectrally encoded microspheres. Lab on a Chip, 12, 4716-4723 (2012).

https://doi.org/10.1039/c2lc40699c

[7] Zhao Y., Zhao X., Tang B., Xu W., Li J., Hu J., Gu Z.: Quantum-dot-tagged bioresponsive hydrogel suspension array for multiplex label-free DNA detection. Advanced Functional Materials, 20, 976-982 (2010). https://doi.org/10.1002/adfm.200901812

[8] Shao Y., Chen H., Zhang W., Zhao Y-J., Fan L-J.: Preparation of monodispersed spectrally encoded microspheres with three different types of fluorophores. Journal of Materials Science, 52, 3906-3916 (2016). https://doi.org/10.1007/s10853-016-0651-8

[9] Liang Y., Abdelrahman A. I., Baranov V., Winnik M. A.: The synthesis and characterization of lanthanideencoded poly(styrene-co-methacrylic acid) microspheres. Polymer, 52, 5040-5052 (2011). https://doi.org/10.1016/j.polymer.2011.08.056

[10] Zhang X., Xiao Y., He L., Zhang Y.: Through-bond energy transfer cassettes for multicolor encoding. Journal of Organic Chemistry, 79, 6315-6320 (2014).

https://doi.org/10.1021/jo500653r

[11] Sarder P., Nehorai A.: Statistical design of position-encoded microsphere arrays. IEEE Transactions on Nanobioscience, 10, 16-29 (2011). https://doi.org/10.1109/TNB.2010.2103570

[12] Lin G., Makarov D., Medina-Sánchez M., Guix M., Baraban L., Cuniberti G., Schmidt O. G.: Magnetofluidic platform for multidimensional magnetic and optical barcoding of droplets. Lab on a Chip, 15, 216-224 (2015). https://doi.org/10.1039/c4lc01160k

[13] Lee J., Bisso P. W., Srinivas R. L., Kim J. J., Swiston A. J., Doyle P. S.: Universal process-inert encoding architecture for polymer microparticles. Nature Material, 13, 524-529 (2014). https://doi.org/10.1038/NMAT3938

[14] He L., Ai C., Wang W., Gao N., Yao X., Tian C., Zhang K.: An effective three-dimensional surface-enhanced Raman scattering substrate based on oblique Si nanowire arrays decorated with Ag nanoparticles. Journal of Materials Science, 51, 3854-3860 (2016). https://doi.org/10.1007/s10853-015-9704-7

[15] Vaidya S., Couzis A., Maldarelli C.: Reduction in aggregation and energy transfer of quantum dots incorporated in polystyrene beads by kinetic entrapment due to cross-linking during polymerization. Langmuir, 31, 3167-3179 (2015). https://doi.org/10.1021/la503251s

[16] Wang Z., Zong S., Li W., Wang C., Xu S., Chen H., Cui Y.: SERS-fluorescence joint spectral encoding using organic-metal-QD hybrid nanoparticles with a huge encoding capacity for high-throughput biodetection: Putting theory into practice. Journal of the American Chemical Society, 134, 2993-3000 (2012).

https://doi.org/10.1021/ja208154m
[17] Zhu Y., Xu H., Chen K., Fu J., Gu H.: Encoding through the host-guest structure: Construction of multiplexed fluorescent beads. Chemical Communications, 50, 1404114044 (2014).

https://doi.org/10.1039/c4cc05793g

[18] Wilson R., Cossins A. R., Spiller D. G.: Encoded microcarriers for high-throughput multiplexed detection. Angewandte Chemie International Edition, 45, 61046117 (2006). https://doi.org/10.1002/anie.200600288

[19] Mathur A., Kelso K. M.: Multispectral image analysis of binary encoded microspheres for highly multiplexed suspension arrays. Cytometry Part A, 77, 356-365 (2010). https://doi.org/10.1002/cyto.a.20841

[20] Peng G., Zhao C., Liu B., Ye F., Jiang H.: Immobilized trypsin onto chitosan modified monodisperse microspheres: A different way for improving carrier's surface biocompatibility. Applied Surface Science, 258, 55435552 (2015).

https://doi.org/10.1016/j.apsusc.2012.01.071

[21] Pang S., Beckham R. E., Meissner K. E.: Quantum dotembedded microspheres for remote refractive index sensing. Applied Physics Letters, 92, 221108/1-221108/3 (2008).

https://doi.org/10.1063/1.2937209

[22] Beier H. T., Coté G. L., Meissner K. E.: Modeling whispering gallery modes in quantum dot embedded polystyrene microspheres. Journal of the Optical Society of America B, 27, 536-543 (2010). https://doi.org/10.1364/JOSAB.27.000536

[23] Ju W., Song X., Yan G., Xu K., Wang J., Yin D., Li L., Qu X., Li Y., Li J.: Layer-by-layer assembly of polyoxometalate-pyrene-decorated fluorescent microspheres for the suspension immunoassay of Listeria monocytogenes. Journal of Materials Chemistry B, 4, 4287-4294 (2016).

https://doi.org/10.1039/c6tb00986g

[24] Mu B., Liu P., Wang A.: Synthesis of polyaniline/carbon black hybrid hollow microspheres by layer-bylayer assembly used as electrode materials for supercapacitors. Electrochimica Acta, 88, 177-183 (2013). https://doi.org/10.1016/j.electacta.2012.10.025

[25] Han B., Choi N., Kim K. H., Lim D. W., Choo J.: Application of silver-coated magnetic microspheres to a SERS-based optofluidic sensor. Journal of Physical Chemistry C, 115, 6290-6296 (2011). https://doi.org/10.1021/jp112265e

[26] You L., Li R., Dong X., Wang F., Guo J., Wang C.: Micron-sized surface enhanced Raman scattering reporter/fluorescence probe encoded colloidal microspheres for sensitive DNA detection. Journal of Colloid and Interface Science, 488, 109-117 (2017).

https://doi.org/10.1016/j.jcis.2016.10.086

[27] Kneipp K., Kneipp H., Itzkan I., Dasari R. R., Feld M. S.: Ultrasensitive chemical analysis by Raman spectroscopy. Chemical Reviews, 99, 2957-2976 (1999). https://doi.org/10.1021/cr980133r 
[28] Martín-Yerga D., Pérez-Junquera A., González-García M. B., Hernández-Santos D., Fanjul-Bolado P.: Towards single-molecule in situ electrochemical SERS detection with disposable substrates. Chemical Communications, 54, 5748-5751 (2018).

https://doi.org/10.1039/c8cc02069h

[29] Sharma B., Frontiera R. R., Henry A-I., Ringe E., van Duyne R. P.: SERS: Materials, applications, and the future. Materials Today, 15, 16-25 (2012). https://doi.org/10.1016/S1369-7021(12)70017-2

[30] Thurn R., Kiefer W.: Structural resonances observed in the Raman spectra of optically levitated liquid droplets. Applied Optics, 24, 1515-1519 (1985).

https://doi.org/10.1364/AO.24.001515

[31] Fan X., Jia X., Liu J., Liu Y., Zhang H., Zhang B., Zhang H., Zhang Q.: Morphology evolution of poly(glycidyl methacrylate) colloids in the 1,1-diphenylethene controlled soap-free emulsion polymerization. European Polymer Journal, 92, 220-232 (2017). https://doi.org/10.1016/j.eurpolymj.2017.03.060

[32] Ding K., Hu B., Xie Y., An G., Tao R., Zhang H., Liu Z.: A simple route to coat mesoporous $\mathrm{SiO}_{2}$ layer on carbon nanotubes. Journal of Materials Chemistry, 19, 37253731 (2009).

https://doi.org/10.1039/b821386k
[33] Grama S., Boiko N., Bilyy R., Klyuchivska O., Antonyuk V., Stoika R., Horak D.: Novel fluorescent poly(glycidyl methacrylate) - Silica microspheres. European Polymer Journal, 56, 92-104 (2014). https://doi.org/10.1016/j.eurpolymj.2014.04.011

[34] Wang C., Zhao M., Li J., Yu J., Sun J., Ge S., Guo X., Xie F., Jiang B., Wujcik E. K., Huang Y., Wang N., Guo Z.: Silver nanoparticles/graphene oxide decorated carbon fiber synergistic reinforcement in epoxy-based composites. Polymer, 131, 263-271 (2017).

https://doi.org/10.1016/j.polymer.2017.10.049

[35] Kneipp K., Wang Y., Kneipp H., Perelman L. T., Itzkan I., Dasari R. R., Feld M. S.: Single molecule detection using surface-enhanced Raman scattering (SERS). Physical Review Letters, 78, 1667-1670 (1997). https://doi.org/10.1103/PhysRevLett.78.1667

[36] Guzatov D. V., Vaschenko S. V., Stankevich V. V., Lunevich A. Y., Glukhov Y. F., Gaponenko S. V.: Plasmonic enhancement of molecular fluorescence near silver nanoparticles: Theory, modeling, and experiment. Journal of Physical Chemistry C, 116, 10723-10733 (2012). https://doi.org/10.1021/jp301598w

[37] Gaponenko S. V.: Effects of photon density of states on Raman scattering in mesoscopic structures. Physical Review B, 65, 140303/1-140303/4 (2002). https://doi.org/10.1103/PhysRevB.65.140303 